Journal of Animal and Veterinary Advances 11 (15): 2741-2746, 2012

ISSN: $1680-5593$

(C) Medwell Journals, 2012

\title{
Analysis of Quasispecies Diversity and Mutations for Highly Pathogenic Porcine Reproductive and Respiratory Syndrome Virus in China Based on ORF5 Genes
}

\author{
Peng Zhao, Cheng-Tai Ma and Zhi-Zhong Cui \\ College of Veterinary Medicine, Shandong Agricultural University, 271018 Taian, China
}

\begin{abstract}
To study the quasispecies diversity and mutations of highly pathogenic porcine reproductive and respiratory syndrome virus emerged in 2006 in China, the ORF 5 gene of a wild HP-PRRSV strain SD061 2 was amplified and the amplified product was cloned into $\mathrm{T}$ vector, all positive clones were sequenced and analyzed with DNA Star software. To exclude sequencing errors, another very conservative gene $O R F 6$ with the similar product length to ORF5 gene was amplified, cloned and sequenced as above. About 60 positive clones of $O R F 5$ gene were obtained, sequenced and analyzed and their nucleic acid sequence homology ranging from $97.7-100 \%$, a total of 78 base mutants between them were found. Among these 60 sequence 17 sequence was quite the same and be determined as the sequence of advantage quasispecies which account for the magerority of SD0612. For the conservative ORF6 gene, 54 positive clones were obtained and their nucleic acid sequence homology ranging from $99.2-100 \%$, among these 54 sequences 46 sequences was quite the same, a total of 8 base mutants were found between them. The GP5 sequence for advantages quasispecies of SD0612 was also compared with other reference GP5 sequence of 20 highly pathogenic PRRSV mutants and they shared only 93.5-98.5\% homology, total 23 mutation points between them was found and some mutations located in the epitopes. This study indicate the quasispecies diversity of SD0612 can be indicated by the sequence diversity of $O R F 5$ gene, the mutations between different HP-PRRSV mutants is also great although they have the same deletions in NSP2 gene.
\end{abstract}

Key words: Porcine syndrome virus, quasispecies mutation, ORF5, ORF6, diversity, pathogenic

\section{INTRODUCTION}

Porcine Reproductive and Respiratory Syndrome (PRRS) is a infectious diseases caused by Porcine Reproductive and Respiratory Syndrome Virus (PRRSV) characterized by a serious reproductive disorders to pregnant sows and serious respiratory symptoms and high mortality rates to piglets. The disease have spread to the world's major pig-raising countries and regions and caused great economic losses to the global pig industry since reported for the first time in 1987 (Benfield et al., 1992; Collins et al., 1992). PRRSV has a single-stranded, positive-sence RNA genome of about $15 \mathrm{~kb}$, encoding nine Open Reading Frames (ORF) and the GP5 proteins encoded by ORF5 is considered as the most important antigen protein, ORF5 mutated quickly in nature and always be considered as the most important reference gene for researching variation and evolution of PRRSV (Conzelmann et al., 1993; Snijder and Meulenberg, 1998; Key et al., 2001).

PRRSV has been on the constant mutation and evolution since the first report, an antypical PRRS characteristics of high fever, high morbidity and high mortality spreads rapidly to the main pig-breeding areas and causing a pandemic outbreak in China in 2006 in a very short period, studies have shown that the main pathogen is highly pathogenic porcine reproductive and respiratory syndrome virus variants (Highly pathogenic PRRSV, HP-PRRSV) characterized by 90-base deletion in NSP2 gene. The variants transmitted to $>10$ provinces with about 400,000 fatal cases in China (Tian et al., 2007; Li et al., 2007). When whole genomes were compared, the results showed that the ORF5 gene of the isolates shared a high degree of homology but not completely homologous even for the strains isolated in the same area (Tian et al., 2007; Li et al., 2007). It is speculated that this may stand for different quasispecies of the PRRSV variants. This study attempts to observe the quasspecies diversity of HP-PRRSV strain SD061 2 by studing the quasspecies of its $O R F 5$ gene.

\section{MATERIALS AND METHODS}

Cell lines and virus: Marc-145 cell lines were used for replication of PRRSV. DMEM, pH7.2GIBCO, USA was used as the basic medium. For the growth medium, $10 \%$ of

Corresponding Author: Zhi-Zhong Cui, College of Veterinary Medicine, Shandong Agricultural University, 271018 Taian, China 
Fetal Bovine Serum (FBS) was added in the basic medium. For the maintaining medium, only $2 \%$ of FBS was added. Penicillin and Streptomycin were added to the medium to 100 ug $\mathrm{mL}^{-1}$ respectively. Cell cultures were kept in an incubator with $5 \% \mathrm{CO}_{2}$ at $37^{\circ} \mathrm{C}$.

PRRSV strain SD0612 was isolated and identified in Marc-145 cells. It was isolated from a swine breeder farms with typical PRRS symptoms and lesions in 2006 (Zhao et al., 2010). It was highly pathogenic and caused $100 \%$ mortality when 2 months old antibody negative were inoculated with it (Fig. 1). Sequence analysis indicated that it has a 30 amino acid deletion in NSP2 as other highly pathogenic strains isolated in that period as reported (Tian et al., 2007; Zhao et al., 2010). PRRSV strain SD061 2 was amplified, tittered and reserved in $-80^{\circ} \mathrm{C}$ freezer for further studies.

Amplification and sequencing of $O R F 5$ and $O R F 6$ gene of HP-PRRSV strain SD0612: Infected cells by SD0612 were harvested when $70-80 \%$ of Marc-145 monolayer demonstrated CPE. The viral genomic RNA was extracted from infected Marc-145 cells by Viral RNA KitOMEGA, USA. ORF5 gene was amplified by RT-PCR with a pair of primers ORF5-F 5'-AGCCTGTCTTTTTG CCATCCCT-3' (relative to 13745 bp-13766 bp of Gen Bank No. AY150564) and ORF5-R 5'-CTTTTGTGGAGCCG TGCTA TC-3' (relative to of GenBank No. AY150564). Procedure and conditions for RT-PCR were following to the report of Han et al. (2009). In order to prove the mutations was not caused by sequencing errors, another very conservative gene ORF6 was amplified by RT-PCR with a pair of primers ORF6-F 5'-GCGGTCGCCCATCATTGTG-3' (relative to 14162 bp-14180 bp of GenBank NO. EF112445) and ORF6-R 5'-GCTGGCCATTCCCCTTCTTTTT-3' (relative to 14829 bp-14850 bp of Gen Bank No. EF112445). The amplification product of $O R F 5$ gene was 682 bp while the amplification product of ORF6 gene was $689 \mathrm{bp}$, the two genes were amplified in the same time and with the same amplification conditions. If the mutation was caused by sequencing errors, they should indicate similar variation regulations. RT-PCR products were purified by E.Z.N.A Gel Extraction Kit(OMEGA, USA) through

Table 1: The reference HP-PRRSV strains of China and it's accession number in Genebank

\begin{tabular}{lcc}
\hline Strain & Genotype & Accession No. \\
AH1 & North America & AB359237 \\
GY & North America & EF990728 \\
LJ & North America & EF990730 \\
TQ & North America & EF990732 \\
HuB & North America & AB359238 \\
JN & North America & EF990729 \\
SD & North America & EF990731 \\
NX06 & North America & EU097706 \\
JX143 & North America & EU708726 \\
SY0608 & North America & EU144079 \\
JX2006 & North America & EU880432 \\
SX2007 & North America & EU880434 \\
HN2007 & North America & EU880437 \\
GD2007 & North America & EU880433 \\
YN2008 & North America & EU880435 \\
XL2008 & North America & EU880436 \\
GS2008 & North America & EU880431 \\
JXA & North America & EF112445 \\
HUN4 & North America & EU213123 \\
WUH3 & North America & EU912573 \\
\hline
\end{tabular}
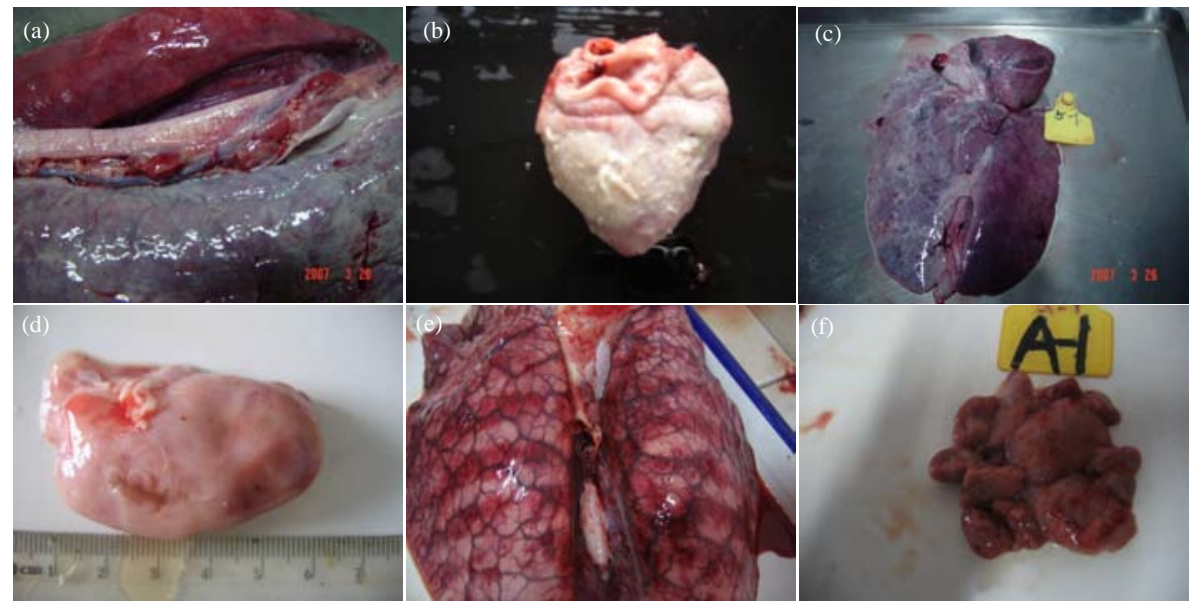

Fig. 1: Pathological changes of dead pig when 2 months old antibody negative were inoculated with SD0612; a, c,e) the typical pathological changes for PRRS can be found in the lung; d, f) Lymph nodes; a-c) the bacterial infection was also found very serious in lung and heart and indicate the infection of HP-PRRSV also caused very serious immunosuppression in pigs 
gel-electrophoresis and cloned into pMD-18T Vector (TaKaRa, Japan). Transformed competent E. coli DH5a colonies were screened and identified. All selected positive clones were sequenced by commecial sequencing service and the sequence data were analyzed by DNA star programs.

Comparisons of $O R F 5$ gene mutations of SD0612 with other reference strains of HP-PRRSV in China: In order to compare the ORF5 sequence of the SD0612 original strain with other highly pathogenic strain of HP-PRRSV in various regions of China, 20 issue ORF5 sequences were used as the reference sequence from GenBank and their accession number was shown in Table 1 .

\section{RESULTS AND DISCUSSION}

The quasispecies diversity of SD0612 based on $O R F 5$ and ORF6 gene: About 60 positive clones for $O R F 5$ gene of SD0612 were sequenced and alalyzed and their nucleic acid sequence homology ranging from $97.7-100 \%$, a total of 78 nucleotide differences between them was found. Among these 60 nucleic acid sequence 17 sequence was quite the same, researchers determined this sequence as the sequence of the advantage quasispecies which account for the magerority of the SD0612. Other quasispecies sequence shared 94.5-99.5\% homology with the advantages quasispecies. For the conservative gene ORF6, 54 positive clones were obtained and their nucleic acid sequence homology ranging from 99.2-100\%, much high than that of ORF5 gene. Among these 54 nucleic acid sequences 46 sequence was quite the same, a total of 8 sites of base differences were found in these sequences. The sequence difference between different clones of ORF5 gene indicate that HP-PRRSV strain SD0612 was composed by a varity of quasispecies, these quasispecies shared a comparative high homology in genome. These quasispecies have some point mutants from each other and these point mutants usually be found in some genes relative to antigen which mutant quickly such as ORF5 gene. While, the quasispecies diversity of PRRSV can not be checked by ORF 6 gene for it is very conservative and only little mutants can be found and analyzed between different quasispecies.

Comparisons of $O R F 5$ gene of SD0612 with other reference strains of $\mathrm{HP}$-PRRSV in China and their mutations: Among these 60 nucleic acid sequences of ORF5 gene of SD0612, 17 sequences was quite the same and researchers determined this sequence as the sequence of the advantage quasispecie. This sequence shared 93.5-98.5\% homology with other reference GP5 sequence of 20 highly pathogenic PRRSV variants. There are 23 mutation points between SD0612 and the reference GP5 sequencea nd the amino acids mutants between them distributed mainly in the following areas in GP5: \#9-16aa, \#25-35aa, \#54-59aa, \#81-89aa, \#1 59-165aa, \#185-188aa, \#196-199aa (Fig. 2).

The porcine reproductive and respiratory syndrome virus is one of the most important pathogen causing porcine reproductive disorders in recent years, PRRSV is on the constant variation and evolution since the first report. GP5 is the major antigen protein of PRRSV and its coding gene ORF5 mutant quickly and always be the most important target for researching the evolution and quasispecies diversity of PRRSV (Conzelmann et al., 1993; Snijder and Meulenberg, 1998; Key et al., 2001).

Because of the lack of corrective mechanism for RNA polymerase, RNA is prone to mutations during duplications. Therefore, a RNA virus strains in the cell culture especially in the same animal body is actually a group composed by different quasispecies whose genomes have some difference. The quasispecies of virus has good correlation with gene duplication. It is speculated the mutation chance of each nucleotide in $H A$ gene of Avian Influenza Virus (AIV) H9N2 subtype is $2 \times 10^{-3}$ for every copy cycle that is about a base change will occur every duplication for H9N2 subtype (Liu et al., 2002). So to observe the quasispecies diversity of PRRSV, researchers can study the quasispecies diversity of some genes of PRRSV, especially for the ORF5 gene which mutant quickly and has good correlation with the antigen. In this study, ORF5 gene of a field HP-PRRSV strain SD0612 was amplified, sequenced and their nucleic acid sequence homology ranging from $97.7-100 \%$, a total of 78 base mutants between them were found while another conservative ORF6 gene, 54 positive clones were obtained and their nucleic acid sequence homology ranging from $99.2-100 \%$, a total of 8 base mutants were found in these sequence. Among these 60 sequences for ORF5 gene, 17 sequences was quite the same, researchers determined this sequence as the sequence of advantage quasispecies which account for the magerority of SD0612 original strain. While for the conservative ORF6 gene, among 54 sequences 46 sequence was quite the same and only 9 kinds of sequence can be found among them, there is no doubt it is because the ORF6 gene is so conservative and the difference between different quasispecies is ignored. From the discussion, researchers can conclude the quasispecies diversity of SD0612 can be indicated by the sequence diversity of ORF5 gene. During the last 10 years, more and more neutralization epitopes on $O R F 5$ gene were identified with neutralizing Monoclonal Antibodies (Mabs) such as residues 36-52 


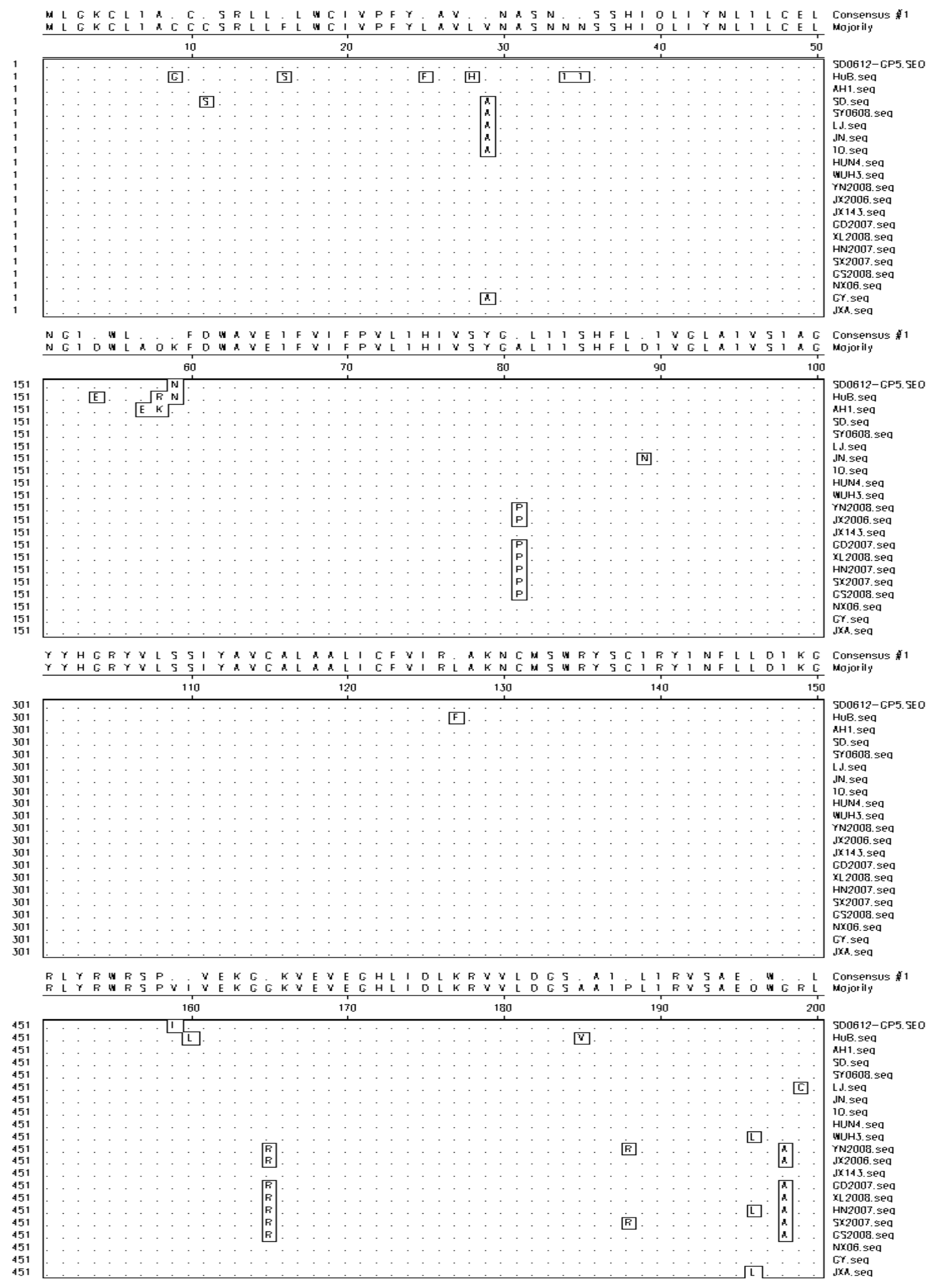

Fig. 2: The alignment of amino acid between the riginal straina and the advantage quasispecies individuals from the group with antibody and HP-PRRSV reference strain in China 
(Plagemann et al., 2002) and 29-35 (Wissink et al., 2003). A proline residue at position 24 of ORF5 may enabled recognition by the neutralizing MAbs. The residues 37-45 was also recognized by pig neutralizing sera. Some other epitopes were also recognized by some non-neutralizing MAbs or mouse sera against recombinant proteins or synthesized peptides such as residues $130-170$ and 170-201 (Rodriguez et al., 2001), residues 146-156, 164-180 and 196-200 (Li et al., 2009; Zhou et al., 2009). In this study, the GP5 sequence for advantages quasispecies of SD0612 was compared with other reference GP5 sequence of 20 highly pathogenic PRRSV variants and they shared only 93.5 and $98.5 \%$ homology. The amino acids mutants between them distributed mainly in the following areas in GP5: \#9-1 6aa, \#25-35aa, \#54-59aa, \#81-89aa, \#159-165aa, \#185-188aa, \#196-199aa (Fig. 2). Researchers can find some amino acids mutants located in the neutralization epitopes on GP5 such as the mutation of \#25-35aa, \#196-199aa. It made us to have an interest to study if antibodies or cell-mediated immunity to PRRSV in vaccinated swine herds will promote antigenic evolution, especially in the GP5 which closely related to virusneutralization.

In the interaction with the host, the virus continues to be in the evolution and mutations. In the past 10 years, much attention was given to the influence of immune selective pressures on evolutions of viruses. In recent years, the laboratory successfully studied effects of immune selective pressures on evolutions of some chicken viruses during passages in cell cultures or chicken embryos with specific antibodies such as Avian Leukosis Virus (ALV) (Wang and Cui, 2006), H9N2 subtype Avian influenza virus (H9-AIV) (Lou et al., 2009), Newcastle Disease Virus (NDV) field strain (Gong and Cui, 2011).

\section{CONCLUSION}

This study shows that in all experiments, effects of antibody-mediated immune selective pressures on evolution of some specific antigen genes related to protective immunity were clearly documented. Researchers have started the continuously passage for SD0612 in Marc-145 cell cultures without or with anti-SD0612 pig sera so to observe the evolution of ORF5 gene and the quasispecies diversity of PRRSV under the immune selective pressures.

\section{ACKNOWLEDGEMENT}

Thanks to the help from president Qu Li-xin of YI-Sheng Animal Disease Research Center.

\section{REFERENCES}

Benfield, D.A., E. Nelson, J.E. Collins, L. Harris and S.M. Goyal et al., 1992. Characterization of Swine Infertility and Respiratory Syndrome (SIRS) virus (isolate ATCC VR-2332). J. Vet. Diagn. Invest., 4: $127-133$.

Collins, J.E., D.A. Benfield, W.T. Christianson, L. Harris and J.C. Hennings et al., 1992. Isolation of swine infertility and respiratory syndrome virus (isolate ATCC VR-2332) in North America and experimental reproduction of the disease in gnotobiotic pigs. J. Vet. Diagn. Invest., 4: 117-126.

Conzelmann, K.K., N. Visser, P. van Woensel and H.J. Thiel, 1993. Molecular characterization of porcine reproductive and respiratory syndrome virus, a member of the arterivirus group. Virology, 193: 329-339.

Gong, Y. and Z. Cui, 2011. Epitope variation in the Newcastle disease virus $\mathrm{HN}$ gene under antibody immune selective pressure in cell culture. Sci. China Life Sci., 54: 474-479.

Han, W., J.J. Wu, X.Y. Deng, Z. Cao and X.L. Yu et al., 2009. Molecular mutations associated with the in vitro passage of virulent porcine reproductive and respiratory syndrome virus. Virus Genes, 38: 276-284.

Key, K.F., G. Haqshenas, D.K. Guenette, S.L. Swenson, T.E. Toth and X.J. Meng, 2001. Genetic variation and phylogenetic analyses of the ORF5 gene of acute porcine reproductive and respiratory syndrome virus isolates. Vet. Microbiol., 83: 249-263.

Li, B., S. Xiao, Y. Wang, S. Xu, Y. Jiang, H. Chen and L. Fang, 2009. Immunogenicity of the highly pathogenic porcine reproductive and respiratory syndrome virus GP5 protein encoded by a synthetic ORF5 gene. Vaccine, 27: 1957-1963.

Li, Y., X. Wang, K. Bo, X. Wang and B. Tang et al., 2007. Emergence of a highly pathogenic porcine reproductive and respiratory syndrome virus in the Mid-Eastern region of China. Vet. J., 174: 577-584.

Liu, H.Q., Y. Huang, J. Cheng, D.X. Peng, L.J. Jia, R.L. Zhang and X.F. Liu, 2002. Genetic mutations of the hemagglutinin gene of $\mathrm{H}_{-} 9 \mathrm{~N} \_2$ subtype avian influenza viruses under the selective pressure of vaccination. Chin. J. Virol., 18: 149-150.

Lou, B.H., X.T. Zhu, B.B. Sun and Z.Z. Cui, 2009. Mutations of the hemagglutinin gene of H9N2 subtype avian influenza viruses under selective pressure of antibody. Acta Microbiol. Sin., 49: 955-959.

Plagemann, P.G.W., R.R.R. Rowland and K.S. Faaberg, 2002. The primary neutralization epitope of porcine respiratory and reproductive syndrome virus strain VR-2332 is located in the middle of the GP5 ectodomain. Arch. Virol., 147: 2327-2347. 
Rodriguez, M.J., J. Sarraseca, J. Fominaya, E. Cortes, A. Sanz and J.I. Casal, 2001. Identification of an immunodominant epitope in the $\mathrm{C}$ terminus of glycoprotein 5 of porcine reproductive and respiratory syndrome virus. J. Gen. Virol., 82: 995-999.

Snijder, E.J. and J.J.M. Meulenberg, 1998. The molecular biology of arteriviruses. J. Gen. Virol., 79: 961-979.

Tian, K., X. Yu, T. Zhao, Y. Feng and Z. Cao et al., 2007. Emergence of fatal PRRSV variants: Unparalleled outbreaks of atypical PRRS in China and molecular dissection of the unique hallmark. PLoS ONE, Vol. 2, No. 6. 10.1371/journal.pone.0000526

Wang, Z.F. and Z.Z. Cui, 2006. Evolution of gp85 gene of subgroup $J$ avian leukosis virus under the selective pressure of antibodies. Sci. China Ser. C: Life Sci., 49: $227-234$.
Wissink, E.H.J., H.A.R. van Wijk, M.V. Kroese, E. Weiland, J.J.M. Meulenberg, P.J.M. Rottier and P.A. van Rijn, 2003. The major envelope protein, $\mathrm{GP}_{5}$, of a European porcine reproductive and respiratory syndrome virus contains a neutralization epitope in its N-terminal ectodomain. J. Gen. Virol., 84: 1535-1543.

Zhao, P., T.C. Ma and Z.Z. Cui, 2010. Evolution of porcine reproductive and respiratory syndrome virus under antibody immune selective pressures. Sci. Sin. Vitae, 40: 952-962.

Zhou, Y.J., H. Yu, Z.J. Tian, J.X. Liu and T.Q. An et al., 2009. Monoclonal antibodies and conserved antigenic epitopes in the C terminus of GP5 protein of the North American type porcine reproductive and respiratory syndrome virus. Vet. Microbiol., 138: 1-10. 